\title{
Variable Numerical-Aperture Temporal-Coherence Measurement of Resonant-Cavity LEDs
}

\author{
Ricardo C. Coutinho, Member, IEEE, David R. Selviah, Member, IEEE, Member, OSA, Rupert F. Oulton, \\ Jonathan W. Gray, Paul N. Stavrinou, Hugh D. Griffiths, Fellow, IEEE, and Gareth Parry
}

\begin{abstract}
The first interferometric measurements of temporalcoherence length variation with numerical aperture (NA) are described for $650 \mathrm{~nm}$, resonant-cavity light-emitting diodes (LEDs) agreeing with spectrally derived results. The interferometrically measured coherence length ( $22 \mu \mathrm{m}$ to $32 \mu \mathrm{m})$ reduced by $37 \%$ for a 0.42 increase in NA. For a larger range of NA $(0-1)$, this would give coherence lengths $(10 \mu \mathrm{m}-40 \mu \mathrm{m})$ lying in the gap between that of conventional LEDs $(\sim 5 \mu \mathrm{m})$ and superluminescent diodes ( $\sim 60 \mu \mathrm{m})$.
\end{abstract}

Index Terms-Coherence modulation, light-emitting diodes, modal noise, optical-coherence length, optical spectra, temporal coherence.

\section{INTRODUCTION}

$\mathbf{T}$ HE TEMPORAL-coherence length of light-emitting diode (LED) optical sources may be used to assess the resolution of low-coherence interferometers, the suitability of the source for use in coherence-modulation communications [1], and the amount of modal, or speckle, noise in multimode fibers [2]. Low-coherence interferometers and coherence-modulated transmission require the use of low-coherence optical sources [1]. Low temporal-coherence sources such as LEDs or multitransverse (spatial) mode vertical-cavity surface-emitting lasers (VCSELs), can reduce the modal, or speckle, noise that would otherwise occur in multimode fibers when using lasers. The optical autocorrelation function or self-coherence interferogram may be conveniently measured using an interferometer and used to calculate the coherence length as a measure of the amount of coherence. The Fourier transform relationship (Wiener-Khintchine theorem [14]) between the self-coherence interferogram $\Gamma(\tau)$ and the power spectrum of a device means that the spectral linewidth of an optical source can be found from the coherence interferogram. However, there seem to be several definitions of coherence length in use by different authors.

A Mach-Zehnder interferometer with a piezoelectric translator was used to measure the modulation depth of the interferogram for $\sim 980 \mathrm{~nm}$ VCSELs [3]. An exponential curve was fitted to the falling modulation depth with path difference, relative to the zero path-difference position, with the coherence

Manuscript received May 30, 2002; revised February 12, 2003.

R. C. Coutinho, D. R. Selviah, and H. D. Griffiths are with the Department of Electronic and Electrical Engineering, University College London, London WC1E 7JE, U.K. (e-mail: d.selviah@ee.ucl.ac.uk).

R. F. Oulton, J. W. Gray, P. N. Stavrinou, and G. Parry are with the Centre for Electronic Materials and Devices, The Blackett Laboratory, Imperial College, London SW7 2BZ, U.K.

Digital Object Identifier 10.1109/JLT.2003.809130 length defined as the $1 / e$ point. The spectral linewidth was then inferred from the coherence length by taking the reciprocal of the coherence time using a $1 / \pi$ proportionality constant.

Temporal and spatial coherence measurements of $8-\mu \mathrm{m}$ and $15-\mu \mathrm{m}$ diameter $\sim 840 \mathrm{~nm}$ VCSELs under dc and pulsed applied currents were measured [4] using a Michelson interferometer and the visibility of the fringes was measured as a function of path difference from the zero path-difference position. Coherence lengths of $75 \mathrm{~cm}$ for single mode (found by exponential fitting of the data) and $0.6 \mathrm{~mm}$ for multimode were obtained. Coherence lengths were also calculated from calculated thermal broadening of spectral measurements in a similar way to [3] and found to be in reasonable agreement with the direct interferometric measurements.

The coherence length of several types of $850-\mathrm{nm}, 10-\mu \mathrm{m}$ diameter VCSEL was measured using a Twyman-Green interferometer [5] to quantify fringe visibility. The curves were normalized to account for nonuniform intensity distributions and beam reflections. The coherence length was defined to be where the visibility dropped by $70 \%$ of the peak value. Single transverse mode VCSELs had been found to have coherence lengths of $30 \mathrm{~cm}$ to $55 \mathrm{~cm}$. The visibility curves developed a number of large sidelobes when the lasers were driven into multitransverse mode operation. Multitransverse mode VCSELs had shorter coherence lengths of $2 \mathrm{~cm}$ to $5 \mathrm{~cm}$. When the VCSELs were modulated, the dynamic chirping reduced the coherence length further to $0.5 \mathrm{~cm}$.

More recently, the coherence length of 1318-nm superluminescent diodes was measured using an automated scanning ( $1 \mu \mathrm{m}$ steps) Michelson interferometer to be $60 \mu \mathrm{m}$ [1]. The coherence lengths of multimode lasers were expected to be in the range $100 \mu \mathrm{m}$ to $500 \mu \mathrm{m}$, but the interferograms had a number of close-in and distant sidelobes and so a practical value for coherence length was difficult to determine [1].

In this paper, we use the technique of a scanning Michelson interferometer to measure the coherence length of another type of LED, the resonant-cavity LED (RCLED) [6]. These are an attractive source for use as reliable, low-cost, efficient emitters for short-distance data communications using multimode fiber, such as plastic-optical fiber (POF) [7], [8], since the devices measured operate in the red wavelength region. These are interesting devices since, unlike conventional LEDs, they have a variable width output spectrum as a function of numerical aperture (NA) [9]-[11]. We present direct interferometric measurements of the temporal-coherence length as a function of NA for a RCLED operating at $650 \mathrm{~nm}$. The RCLED device structure 


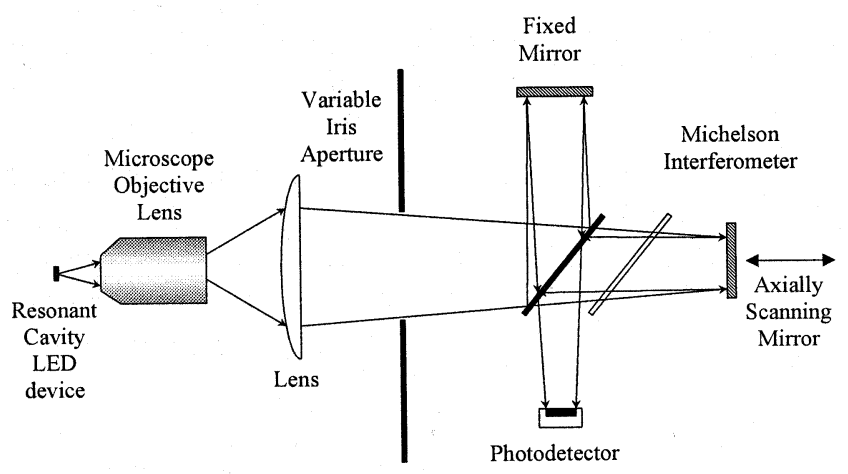

Fig. 1. Experimental coherence interferogram measurement system.

is one of a series described and characterized in earlier reports, where it is the one referred to as being a 4-nm detuned structure [10]-[12].

We also examine two ways of defining the coherence length. Some definitions have been observed to have problems when the autocorrelation function has sidelobes or is very noisy [1]. This may not matter if one is only performing relative measurements. However, in this paper, we also make a comparison with the coherence lengths calculated from the measured power spectra via the Wiener-Khintchine theorem for confirmation. Additionally, most authors use various fixed-inverse relationships to relate coherence length and linewidth which depend on assumptions about spectral lineshape. We avoid this by calculating coherence lengths directly from their self-coherence interferograms for both the direct interferometric measurements and for those calculated by the Wiener-Khinchin theorem from spectral measurements.

\section{TEMPORAL-COHERENCE LENGTH INTERFEROMETRIC MEASUREMENT}

Coupling optics (which allow the input system NA to be controlled), followed by a scanning Michelson interferometer with an output silicon photodetector, were used to record interferograms from the $650 \mathrm{~nm}$ RCLEDs.

To measure the coherence length as a function of NA, the NA of the coupling optics needs to be easily variable (Fig. 1). The coupling optics from the RCLED to the interferometer consist of a $45 \times, 0.65 \mathrm{NA}$ (quoted in the datasheet), microscope objective, a 50-mm diameter, 62.9-mm focal-length plano-convex lens, and a $50-\mathrm{mm}$ variable iris aperture. A variable NA is obtained by inserting the variable iris between the plano-convex lens and the interferometer to transmit a beam with a diameter varying from $1.5 \mathrm{~mm}$ to $25 \mathrm{~mm}$. The small source is positioned in the focal plane of the objective to give the highest degree of collimation: a divergence of $\sim 1.5^{\circ}$ at best due to the lateral extent of the source. The iris is widened to the edge of the beam and this is taken to correspond to the measured NA of the objective lens, NA $=0.53$. Assuming that changes in the output pupil are proportional to changes in the input pupil of the objective, then the NA can be calculated from the iris diameter. This has a similar effect to that of placing a virtual iris between the RCLED and the microscope objective.

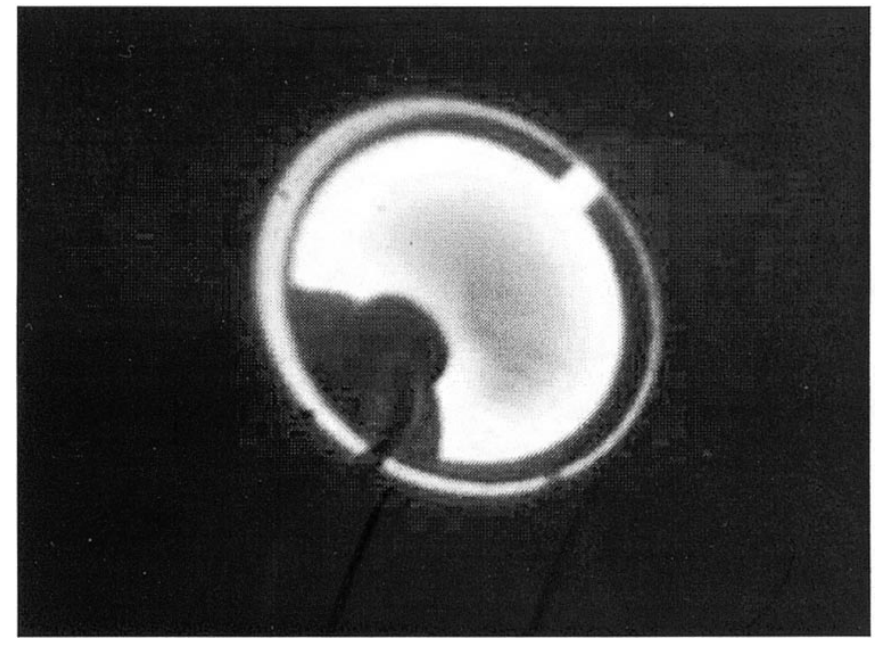

Fig. 2. Illuminated RCLED recorded through the interferometer with the moving mirror blocked, using a CCD camera replacing the photodetector.

As the maximum NA of the microscope objective was important for scaling all of the results, we decided not to rely only on the values quoted in its datasheet. By passing an $\mathrm{He}-\mathrm{Ne}$ laser beam through a rotated microscope objective, the range of angles over which the transmitted beam was extinguished was recorded. The spot passing through the objective lens fell on a screen and it was observed that the image gradually reduced being cut off from one side. The onset of beam cutoff occurred at NA $=0.46$ and continued until NA $=0.6$ due to the finite beam width, so the effective NA was taken to be the mid point of this range, $\mathrm{NA}=0.53$ and the range was marked by horizontal error bars in subsequent graphs having NA as the abscissa.

The separation of the microscope objective and the source was adjusted to image the RCLED onto the full 10-mm diameter, silicon photodetector area through a scanning Michelson interferometer. This reduced any nonrepeatability due to the photodetector's spatial and angular response. In order to set up the imaging through the interferometer it was most convenient to replace the photodetector by a CCD camera. Each mirror was blocked in turn. Fig. 2 shows the image when the moving mirror was blocked. Fig. 2 clearly shows the circular nature of the device and its ring-like electrode, and the shadow of the bonding pad and bond wire. It is similar to photos taken directly through a microscope objective without the interferometer path. The darker region furthest from the ring electrode is caused because all of the current does not spread from the contacts across the device face. Actually, this device is rather large for a typical LED having a $400 \mu \mathrm{m}$ diameter, although it is very convenient for performing photocurrent measurements. We have observed that in 200- $\mu \mathrm{m}$ diameter devices, the uniformity of illumination improves and we would expect any nonuniformity to disappear for smaller area devices. Care had to be taken in alignment to reduce spurious reflections from the beamsplitter which could give rise to an additional faint laterally displaced image. The beams travelling the two paths had similar intensities.

When both mirrors were unblocked, an interferometric image of the device is recorded on the camera. Where the two images of the device overlap a set of fringes is observed. The system was aligned to overlap the two images exactly and to maximize 


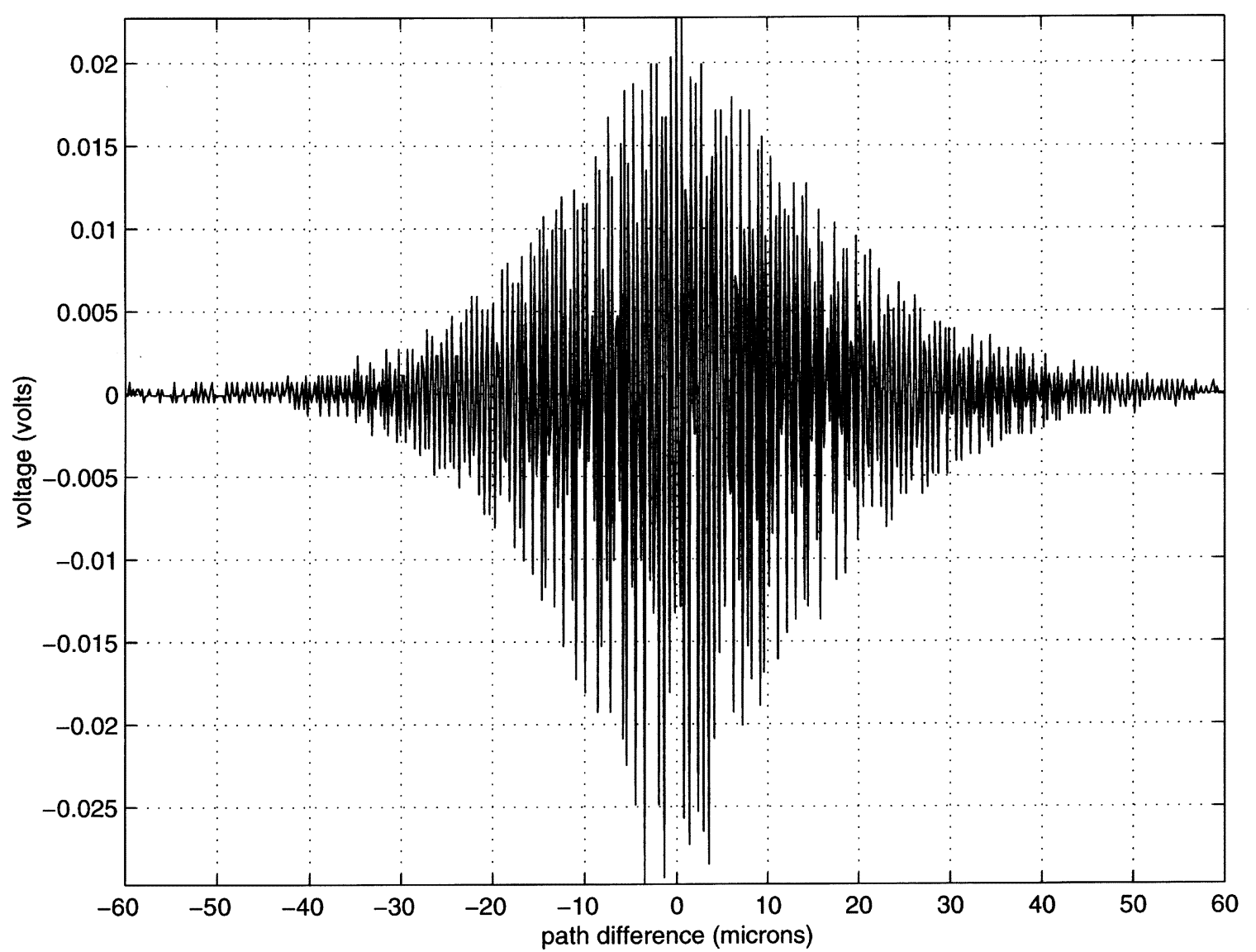

Fig. 3. Recorded interferogram NA $=0.53, I=40 \mathrm{~mA}$, and $400 \mu \mathrm{m}$ aperture.

the fringe spacing at the photodetector plane so that it was of at least the same order as the detector diameter. Ideally, an interferometric image similar to that in Fig. 2 is again obtained whose brightness oscillates as one interferometer mirror is moved to alter the path difference. The CCD camera is then replaced by the photodetector in the same plane so that measurements can be taken.

One interferometer mirror was moved axially at $50 \mu \mathrm{m} / \mathrm{s}$ by a motorized translation stage through $150 \mu \mathrm{m}$ to produce interferograms with a path difference range of $300 \mu \mathrm{m}$. The use of the translation stage allows sufficiently long scans to be performed to encompass the coherence lengths being measured. The translation stage moved in discrete $0.1 \mu \mathrm{m}$ steps generating a spurious stepping frequency during a scan. The stepper motor also generated electromagnetic interference which was difficult to separate by filtering from the fringe carrier as its frequency was close to the fringe carrier frequency. We found that when we replaced the translation stage with a piezoelectric transducer for a different experiment, that due to its higher speed $(21.6 \mathrm{~mm} / \mathrm{s})$ and continuously smooth, although nonlinear, travel, the fringe carrier frequency was well separated from the noise spectrum and so they could be separated by filtering. However, it could not be used in these experiments, as the scan range was insufficient for the coherence length of the RCLEDs. We assessed any misalignment which might occur between the two interferometer mirrors with path difference by using a visible laser source to generate fringes and derived a correction factor which actually had little influence on the results and so was not used.
After amplification, the electrical (interferogram) signal generated from the photodetector was ac coupled (to remove the dc background) to a digital-sampling oscilloscope with a sampling rate of $256 \mathrm{~Hz}$. The signal was then transferred to a computer via a GPIB interface. A 400- $\mu \mathrm{m}$ mesa RCLED device was measured with applied supply currents of 5, 20, and $40 \mathrm{~mA}$, and a 200- $\mu \mathrm{m}$ mesa RCLED device with currents of $5 \mathrm{~mA}$ and $20 \mathrm{~mA}$ to investigate whether finite-source-size effects were of importance for these measurements and to investigate the spectral stability of the output spectrum with increasing current. For each NA, five interferograms were recorded and used to find the average coherence length and standard deviation.

The zero path-difference position was found by using a wideband white-light halogen source to plot its double-sided interferogram, from which the position of the peak at which all wavelengths add constructively could easily be determined as it is somewhat different from the neighboring peaks.

\section{SignAL-PROCESSING AND COHERENCE-LENGTH CALCUlation}

One hundred and seventy five double-sided interferograms, each having 1024 samples, were acquired and processed (Fig. 3). Double-sided interferograms include both positive and negative path-length differences, doubling the number of data points. Depending on which definition of coherence length is used this can reduce the error due to accurate determination of the zero path difference position. Any residual dc component 


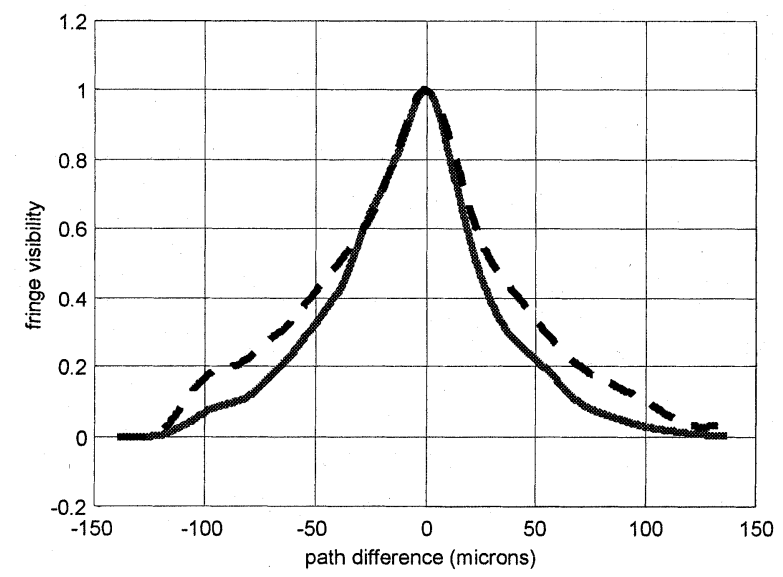

Fig. 4. Comparison of filtered interferograms acquired with the largest NA (inner continuous curve) and smallest NA (outer broken curve).

was removed and the absolute value of the fringe profile found before lowpass filtering to obtain the interferogram envelope. The filter used was a fifth-order Butterworth low-pass filter due to its maximally flat amplitude response and ease of implementation. The filter bandwidth was chosen to remove both the fringe carrier and a small modulation artifact due to the nonuniform velocity of the motorized translation stage (Fig. 4). This was done by first calculating the Fourier transform (using an FFT) of the unfiltered interferogram. The cutoff frequency could then be selected to remove the fringe carrier and high-frequency noise, while preserving the low-frequency envelope. Fig. 4 shows two filtered interferograms yielding their envelopes, for the largest and smallest NAs.

Some of the interferograms had large sidelobes, which could lead to problems in some ways of defining coherence length. An automatic way of calculating the coherence length without the need for user interpretation of the envelopes was required. We investigated two possible formulae for coherence length that do not appear to have been used by recent authors. [13] gives the coherence length to be

$$
\Delta \tau_{M}=\int_{-\tau_{\max }}^{\tau_{\max }}|\Gamma(\tau)|^{2} d \tau
$$

In [14], the coherence length $\Delta \tau$ is found by calculating the normalized root mean square (RMS) width of the squared modulus of the self-coherence function $\Gamma(\tau)$

$$
(\Delta \tau)^{2}=\frac{\int_{-\tau_{\max }}^{\tau_{\max }} \tau^{2} \cdot|\Gamma(\tau)|^{2} d \tau}{\int_{-\tau_{\max }}^{\tau_{\max }}|\Gamma(\tau)|^{2} d \tau}
$$

where $\tau$ is taken to be the path difference in length units. The latter equation assumes that spatial and temporal coherence can be assessed separately in the experiment.

It was found that the cutoff frequency of the software low-pass filter had a strong influence on the coherence lengths found using (1) and almost no effect when using (2) to find the coherence lengths. Equation (2) calculates the coherence length as the RMS width of the self-coherence function, which does not vary with the presence or absence of the fringe carrier in the filtered signal. Equation (1) calculates the coherence length

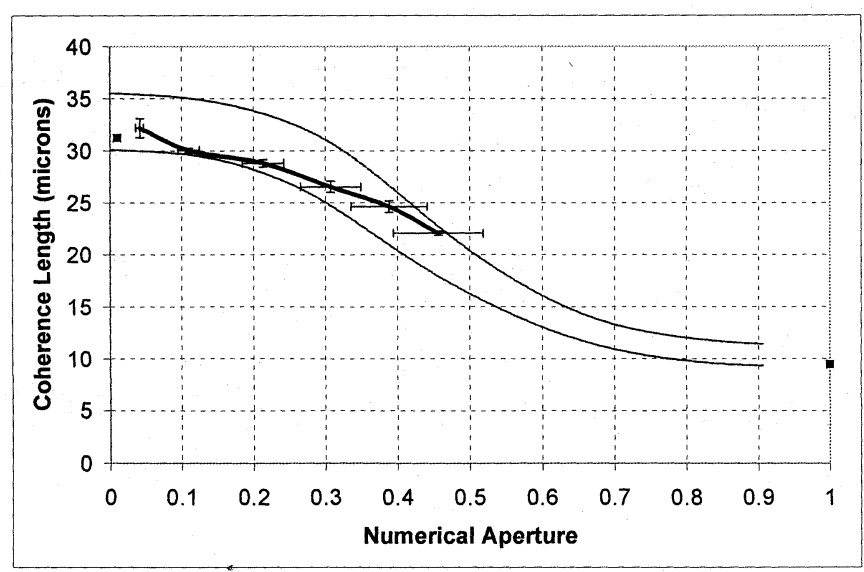

Fig. 5. Coherence length versus NA, $I=20 \mathrm{~mA}, 400 \mu \mathrm{m}$ aperture. Interferometric method (thick line with error bars). Spectrally derived, Gaussian fit interpolation (upper thin curve). Spectrally derived, Lorentzian fit interpolation (lower thin curve). Spectrally derived without interpolation or integration (squares).

by finding the integrated total energy in the self-coherence function, which increases as the dips in the self-coherence function are filled by filtering out the fringe carrier. Equation (2) generally gives somewhat shorter (by about a factor of 2) coherence lengths than (1). Equation (1) gave a greater variation from one measurement to the next of the same device, due to noise, than (2). So we concluded that (2) is a more robust estimate of the coherence length and would be more suited to give reproducible results in an automated system and so was used throughout the following work. However, (2) assumes cross-spectral purity, that the spectral content of the light does not vary across the beam and this remains to be verified.

For each NA, five interferograms were used to deduce five values of coherence length, which were then averaged with the standard deviation plotted as a vertical error bar in Fig. 5.

\section{Coherence-Length Calculations From SPECTRAL MEASUREMENTS}

To validate the interferometric measurements, the coherence length was also calculated from spectral measurements taken using a monochromator with a spectral resolution of $0.1 \mathrm{~nm}$ through a pinhole, which gave a very small NA of less than 0.01 . The device was mounted on a rotation stage and rotated so that the monochromator sampled the emission at several angles from the device surface normal. The spectrally resolved emission profile was measured for a range of angles and wavelengths at a very small angled NA, determined by the size of the pinhole placed in front of the monochromator. This sampled the radiation in a small circular solid angle at an angle to the surface normal. Integration of the spectrally resolved radiation collected within the observation small solid angle, in a circle around the surface normal gives the spectrum emitted into an annulus around the surface normal. These spectra were calculated at discrete angles to the surface normal. Spectra at intermediate angles were interpolated by fitting either Gaussian or Lorentzian curves to the spectral lineshapes. The annuli were integrated over a certain solid angle to give the power spectral 
density at that NA [11]. The reader may find further details of this process in [11]. The spectra were Fourier transformed to obtain the self-coherence function and then the coherence length was found using (2).

Fig. 5 shows the coherence length curves obtained after interpolation using Lorentzian or Gaussian curves to fit the spectral lineshape. Two points are shown on Fig. 5 which did not require such interpolation and integration. The point at $\mathrm{NA}=0.01$, corresponded to the spectral measurement with the pinhole on axis. The point at NA $=1$ corresponds to a spectral measurement of the total light emitted when the device was placed in an integrating sphere. For clarity, no error bars are shown for any of the spectrally derived data although the separation of the two curves indicates the sensitivity to the curve fit used for interpolation.

\section{RESUlts}

The variation of coherence length with NA for the $400-\mu \mathrm{m}$ diode, at $20 \mathrm{~mA}$, is shown in Fig. 5. Within statistical error, no difference was found in coherence length between a point at maximum $\mathrm{NA}=0.53$ and another point taken at $\mathrm{NA}=0.46$. This suggests that from NA $=0.46$ upward very little additional light is collected by the objective and is in agreement with the minimum of the limiting NA of the objective lens measured. The point at maximum NA suffers the effects of the limiting NA of the objective and so has been omitted from Fig. 5.

The interferometric curve lies between, in agreement with, and shows the same trend as the two curves derived from spectral measurements, which in turn agree with the two spectrally derived points obtained without integration or interpolation. The agreement between experimental results is reasonable considering that the measurements were taken using different instruments. The coherence length decreases with increased collection angle or NA, as the spectral linewidth broadens [9], [10].

On closer inspection, the two noninterpolated points obtained from spectral measurements, which did not involve any assumptions, appear to agree more closely with the curve derived by Lorentzian curve fitting. We also found that the Lorentzian curve tended to fit the spectral curves more closely than the Gaussian curve.

The interferometric experimental curve appears to move from the Lorentzian curve to the Gaussian curve at larger NAs, however, due to the size of the horizontal error bars this cannot be conclusively stated. Probably the spectral lineshape is closer to being a Voigt profile, being the convolution of a Gaussian and a Lorentzian and their relative weights change with NA with a stronger weighting toward the Lorentzian. In further work, it will be important to reduce the uncertainty in the NA of the microscope objective (which affects the horizontal error bars of all readings) by characterizing the mapping of the NA from the input of the microscope objective lens to the output.

The coherence length was also found to decrease with increases in current from $5 \mathrm{~mA}$ to $40 \mathrm{~mA}$, by $3 \%$ for low NAs and by $6 \%$ for high NAs corresponding to an increasing linewidth with current. No difference was found between the $400-\mu \mathrm{m}$ and the $200-\mu \mathrm{m}$ diodes for the same NAs and currents to within experimental error. The coherence lengths calculated using (2) were found to be inversely proportional to the spectral linewidths.

\section{CONCLUSION}

The first direct interferometric measurements of coherence length with variable NA for RCLEDs are described and agree with indirect spectroscopic measurements. The interferometric method yields immediate, accurate, direct measurements of the coherence length using a fairly readily constructed experimental setup. It can also be used to assess the changes in spectral linewidth without the need for a spectrum analyzer. Similar coherence results can be derived from spectroscopic measurements although several assumptions are required and some further calculations. The interferometric measurement technique has the advantage of presenting a metric of spectral linewidth that does not depend on wavelength, and of allowing the use of large collection angles.

These results show that the optics used to couple an RCLED to an optical fiber should be carefully designed to achieve a specific NA, which implies a specific coherence length or spectral linewidth. The coherence length dependence on NA must be taken into account in the design of optical systems sensitive to coherence or linewidth when RCLEDs are used as the light source. So when used with multimode fibers, the coherence length reductions with NA will enhance the reductions in modal noise with NA due to the increased number of fiber modes launched [2]. However, when RCLEDs are used with POF, the spectral response of the POF may act as a filter narrowing the spectral linewidth of the light emerging from the fiber. This in turn will result in a lengthening of the coherence length. Since the amount of filtering caused by the fiber depends on its length, the coherence length will also vary with distance, and it is the coherence length of the light emerging from the fiber that will be important for determining modal noise.

RCLED coherence lengths were measured directly to be in the range $22 \mu \mathrm{m}$ to $32 \mu \mathrm{m}$ and were found from spectral measurements to lie between about $10 \mu \mathrm{m}$ and $40 \mu \mathrm{m}$ (corresponding to spectral linewidth changes from about $13 \mathrm{~nm}$ to $5 \mathrm{~nm}$ ). This range lies between the coherence lengths of ultrabright InGaAlP $650 \mathrm{~nm}$ LEDs $(5.44 \mu \mathrm{m})$, which had also been measured directly using the same apparatus, and the coherence length of superluminescent diodes $(60 \mu \mathrm{m}$ using a different definition) [1], so filling an important gap in the coherence spectrum.

\section{ACKNOWLEDGMENT}

The authors would like to thank D. Wickramasinghe, Dstl Portsdown West, for providing some of the experimental apparatus, Prof. H. French for advice, and the Brazilian Navy for the sabbatical leave of R. C. Coutinho.

\section{REFERENCES}

[1] C. Gutiérrez-Martínez, B. Sánchez-Rinza, J. Rogríguez-Asomoza, and J. Pedraza-Contreras, "Automated measurement of optical coherence lengths and optical delays for applications in coherence-modulated optical transmissions," IEEE Trans. Instrum. Meas., vol. 49, pp. 32-36, Jan. 2000 
[2] J. M. Senior, Optical Fiber Communications: Principles and Practice, 2nd ed. Englewood Cliffs, NJ: Prentice-Hall, 1992, pp. 119-121.

[3] R. S. Geels, S. W. Corzine, and L. A. Coldren, "InGaAs vertical-cavity surface-emitting lasers," IEEE J. Quantum Electron., vol. 27, pp. 1359-1367, June 1991.

[4] J. L. A. Chilla, B. Benware, M. E. Watson, P. Stanko, J. J. Rocca, C. Wilmsen, S. Feld, and R. Leibenguth, "Coherence of VCSELs for holographic interconnects," IEEE Photon. Technol. Lett., vol. 7, pp. 449-451, May 1995.

[5] S. A. Kemme, R. K. Kostuk, and C. K. Y. Chun, "Dynamic coherence measurements of index- and gain-guided VCSELs," IEEE Photon. Technol. Lett., vol. 9, pp. 554-556, May 1997.

[6] E. F. Schubert, N. E. J. Hunt, R. J. Malik, M. Micovic, and D. L. Miller, "Temperature and modulation characteristics of resonant-cavity lightemitting diodes," J. Lightwave Technol., vol. 14, pp. 1721-1729, July 1996.

[7] K. Streubel and R. Stevens, " $250 \mathrm{Mbit} / \mathrm{s}$ plastic fiber transmission using $660 \mathrm{~nm}$ resonant cavity light emitting diode," Electron. Lett., vol. 34, no. 19 , pp. $1862-1863,1998$.

[8] M. Pessa, M. Guina, M. Dumitrescu, I. Hirvonen, M. Saarinen, L. Toikkanen, and N. Xiang, "Resonant cavity light emitting diode for a polymer optical fiber system," Semicond. Sci. Technol., vol. 17, pp. R1-R9, June 2002.

[9] P. N. Stavrinou, M. Whitehead, G. Parry, and C. C. Button, "Angular spectrum of visible resonant cavity light-emitting diodes," J. Appl. Phys., vol. 86, no. 6, pp. 3475-3477, 1999.

[10] J. W. Gray, R. F. Oulton, P. N. Stavrinou, M. Whitehead, G. Parry, G. Duggan, R. C. Coutinho, and D. R. Selviah, "Angular emission profiles and coherence length measurements of highly efficient, low voltage resonant cavity light emitting diodes operating around $650 \mathrm{~nm}$," in Light-Emitting Diodes: Research, Manufacturing and Applications V, H. Walter Yao and E. Fred Schubert, Eds. Philadelphia, PA: SPIE, vol. 4278, pp. 81-89.

[11] R. F. Oulton, J. W. Gray, P. N. Stavrinou, and G. Parry, "Insight into planar microcavity emission as a function of numerical aperture," Opt. Commun., vol. 195, pp. 327-338, 2001.

[12] — , "High efficiency, low voltage resonant cavity light emitting diodes operating around $650 \mathrm{~nm}$," Electron. Lett., vol. 36, pp. 1730-1731, Sept. 2000.

[13] I. Mandel and E. Wolf, "Coherence properties of optical fields," Rev. Modern Phys., vol. 37, pp. 231-285, 1965.

[14] M. Born and E. Wolf, Principles of Optics, 6th ed. Cambridge, U.K.: CUP, 1999, p. 540

Ricardo C. Coutinho (M'99) graduated from the Brazilian Naval Academy, Rio de Janeiro, Brazil, as a Navy Officer in 1985. He received the M.Eng. from the University of São Paulo, São Paulo, Brazil, and is currently pursuing the $\mathrm{Ph} . \mathrm{D}$. degree at University College London, London, U.K

He was a Fleet Officer for two years in a frigate, and worked for the Navy's Electronic Repair Center, Rio de Janeiro, Brazil, for five years as the Head of the Sensors Group and two years as the Production Manager. He is currently a Specialist in electro-optics for the Navy's Weapon Systems Directorate, Rio de Janeiro, Brazil. His previous research includes work with spread spectrum communications. His research interests are coherence properties of light, interferometric detection systems, testing and modeling of electro-optical systems, and infrared countermeasures.

Lt.Cdr. Coutinho is a Member of the International Society for Optical Engineers (SPIE) and the Institution of Electrical Engineers (IEE)
David R. Selviah (M'01) received the B.A. and M.A. degrees from Trinity College, Cambridge University, Cambridge, U.K., in 1980 and 1984, respectively, studying natural sciences and specializing in physics and theoretical physics.

He carried out research into surface acoustic wave devices at the Allen Clark Research Centre, Plessey (Caswell) Ltd., U.K. (now Bookham Technology plc, Caswell, U.K.) for three years. Thereafter, he worked and studied as a Research Fellow with the Department of Engineering Science, Oxford University, Oxford, U.K., for over three years. In 1987, he joined his present employer, the Department of Electronic and Electrical Engineering, University College London, London, U.K., as Research Fellow, and was appointed to a Lecturer post in 1989. He has 80 publications of various types. His research interests include optical devices and systems, optical interconnects, pattern recognition, neural networks, and low-cost, high-bit rate short optical-fiber links.

Dr. Selviah is a Member of the Institute of Physics, the Optical Society of America (OSA), and the European Optical Society.

Rupert F. Oulton, photograph and biography not available at the time of publication.

Jonathan W. Gray, photograph and biography not available at the time of publication.

Paul N. Stavrinou, photograph and biography not available at the time of publication.

Hugh D. Griffiths (M'86-SM'90-F'99) received the M.S. degree from the University of Oxford, Oxford, U.K., in 1978, and the Ph.D. and DSc.(Eng.) degrees from University College London, London (UCL), U.K., in 1986 and 2000, respectively.

He is currently Head of the Department of Electronic and Electrical Engineering, UCL. His research interests include radar and sonar systems and signal processing, and antenna measurement techniques. He has published over 250 papers and technical articles in the fields of radar, sonar, and antennas.

Prof. Griffiths received the IEEE AESS Fred Nathanson Award in 1996 and was elected to a Fellowship of the Royal Academy of Engineering in 1997.

Gareth Parry, photograph and biography not available at the time of publication. 